\title{
Optimization of active magnetic bearings for automotive flywheel energy storage systems based on soft magnetic materials
}

\author{
M. Recheis ${ }^{1}$, B. Schweighofer ${ }^{1}$, P. Fulmek ${ }^{2}$ and H. Wegleiter ${ }^{1}$ \\ ${ }^{1}$ Institute of Electrical Measurement and Measurement Signal Processing, Graz University of Technology, Graz, Austria \\ ${ }^{2}$ Institute of Sensor and Actuator Systems, Vienna University of Technology, Vienna, Austria
}

\begin{abstract}
For active magnetically suspended rotors in mobile flywheel energy storage systems the lowest possible weight, smallest size and a low price is required. Since the flywheel is operated in vacuum and very little heat can be dissipated from the rotor, the bearing's magnetic losses have to be as minimal as well. This paper compares the design and optimization of homopolar radial active magnetic bearings with 3 different types of laminated steel. The first type is a standard transformer steel, the second one is high flux cobalt steel and the third one is high flux cobalt steel with high tensile strength.
\end{abstract}

\section{Introduction}

In order to achieve highest possible energy densities, the flywheel is constructed from materials with high tensile strength and low weight. This leads to high rotational speeds and therefore requires magnetic bearings and operation in vacuum [1]. Since losses in the flywheel's rotor can only be radiated, the magnetic losses of the electric machine and the magnetic bearings should be as small as possible. Therefore this paper is presenting the optimization of homopolar active magnetic bearings. A comparison of measured rotor losses for homopolar and heteropolar magnetic bearings can be found in [2].

Ding et al. [3] present a finite element method (FEM) based optimization. The magnetic force is calculated with Maxwell's stress tensor.

Stumberger et al. also [4] present an optimization technique using FEM. Since the objective function is not continuous, they use the differential evolution algorithm. The discretization of the number of windings and the number of steel sheets in the bearing's lamination stack result in a discontinuous objective function. Even though the error space has a global minimum, it is not easy to find with a conventional gradient method.

\section{Motivation}

To facilitate the integration of the flywheel energy storage into a passenger car, the design height should be as low as possible. Therefore the width to height ratio of the radial bearing is set to one half. The most frequent and severe bearing loads are oriented in driving direction and are caused by means of braking and accelerating. The centrifugal reaction torque is oriented perpendicular to the deflection. Therefore four radial bearings are optimal. To find a weight dependent optimum for different kinds of laminated steel, it is necessary to compare a lot of optimization results. The calculation time and calculation effort of FEM models require an alternative approach. Therefore we decided to build up an equivalent magnetic circuit model to compute the mean flux density and the magnetic force to obtain the cost function.

\subsection{Circumventing the finite element method}

As Sun et al. present in [5] the equivalent circuit model contains the main iron flux-path with $R 1, R 3$ and $R 5 . R 1$ represents the left half of the $\mathrm{u}$-core in figure $2 . R 3$ represents the right side of the $\mathrm{u}$-core and $\mathrm{R} 5$ represents the rotor. The surrounding air at the limbs causes the edge effect. The air gap and the surrounding air are both included into $R 4$. The winding area's stray flux path is represented by $R 2$.

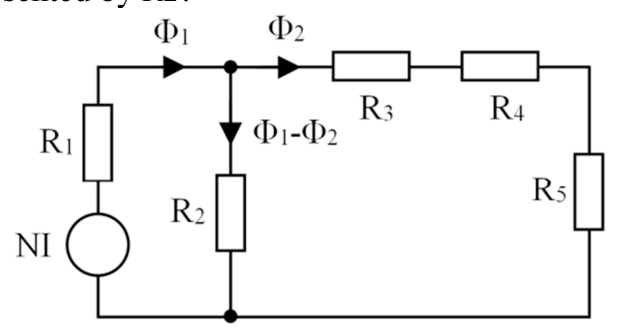

Fig. 1: Equivalent circuit diagram

The reluctances $R 1$ to $R 5$ are computed with (1). The length is represented by $l_{i}, A_{i}$ is the cross-sectional area and $\mu_{R i}$ is the relative permeability. Since the values of $R_{i}$ 
are dependent on the relative permeability $\mu_{R i}$, they have to be updated within each iteration.

$$
R_{i}=\frac{l_{i}}{\mu_{0} * \mu_{R_{i}} * A_{i}}
$$

To be more accurate and to adjust the equivalent circuit model ECM to the FEM results, the four parameters depicted in figure 2 are introduced.

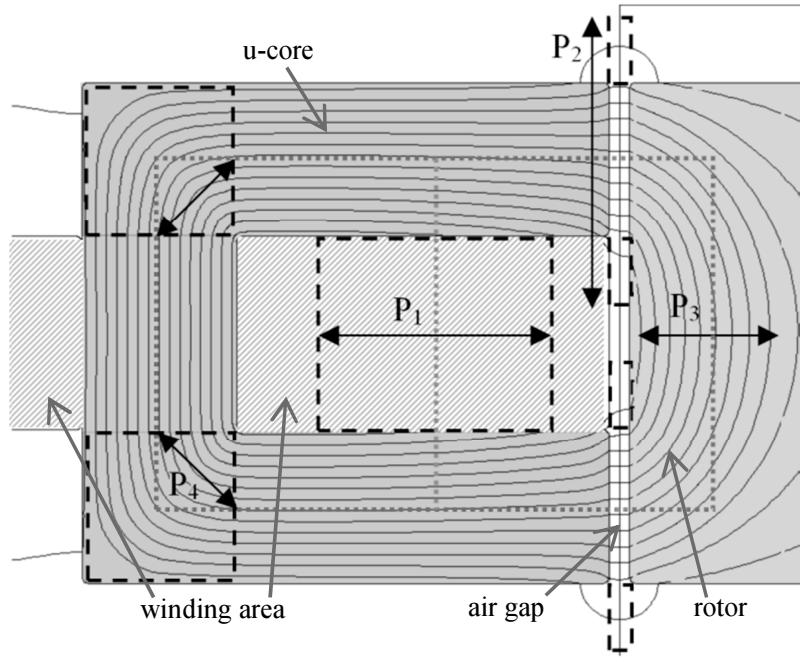

Fig. 2: Parameters to adjust the ECM

Parameter Pl scales the effective width of the winding area to adjust the amount of stray flux that short-circuits the magnetic circuit through the bearing's windings. $P 2$ scales the air gap width to adjust the edge effect. Due to the edge effect the air gap is widened, which reduces the reluctance of the air gap and also reduces the magnetic flux density in the air gap. Parameter $P 3$ adapts the effective penetration depth of the magnetic field in the radial bearing's rotor, which is thicker than the bearing's limbs. $P 4$ scales the effective iron length of the edges.

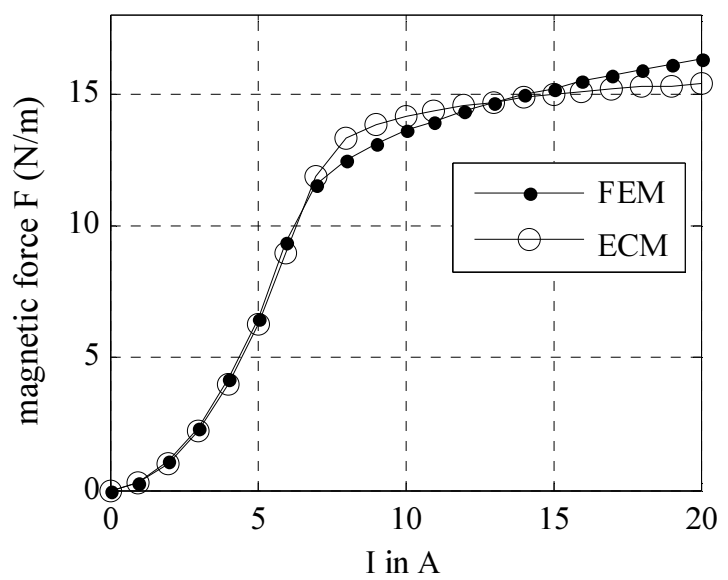

Fig. 3: Comparison of the calculated magnetic force

The range of the magnetic bearing's current in figure 3 was chosen from 0 to 20 amperes, to obtain the relatively high accuracy of the EC model in the area of 15 amperes. This is the maximum current for which the magnetic bearing will be optimized later. The weight and cost dependent curves in figure 6 and 8 are also optimized at 15 ampere. The relative error at 15 ampere is about $1.5 \%$, and the mean relative error is $3.6 \%$. Figure 4 shows the magnetic flux through the air gap. Again, like in figure 3 the model is accurate in the first third of the current range. The relative error of the EC model at 15 amperes is about $1.5 \%$.

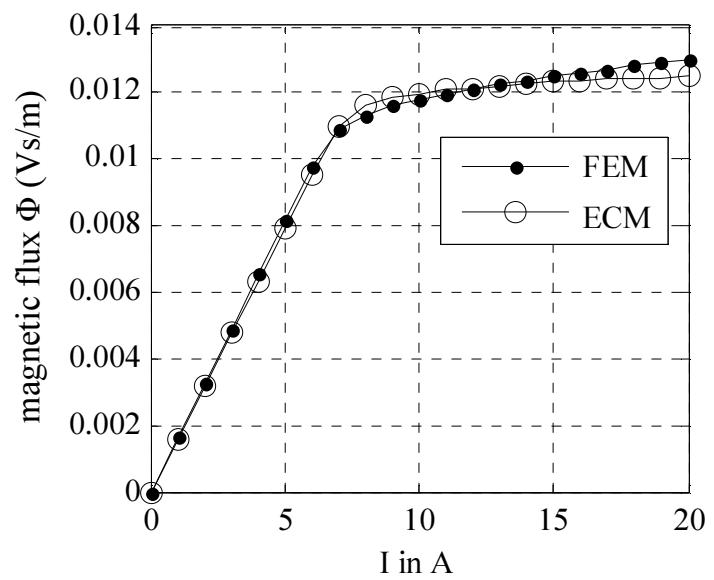

Fig. 4: Comparison of the magnetic flux through the air gap

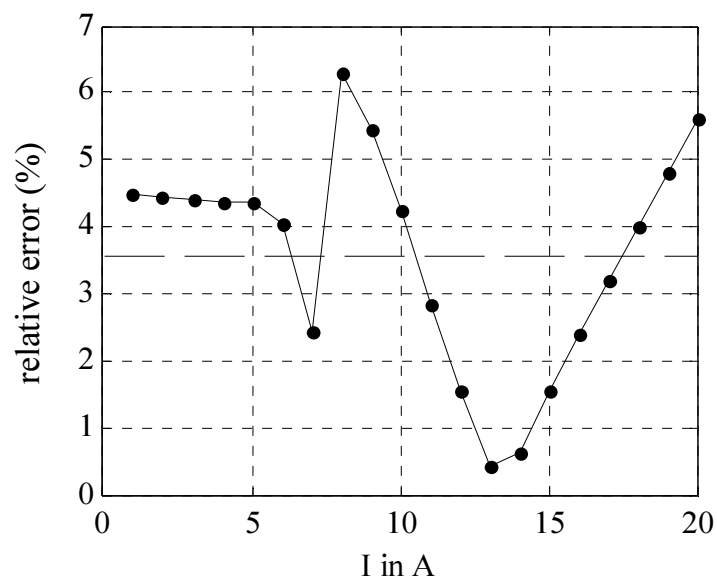

Fig. 5: Relative error of the EC model

The advantage of the EC model is that one whole optimization process can be completed in about a second. Therefore a comparison of different optimization results is achievable as mentioned in section 2 . The main input into the FEM and the ECM are the geometries, the magnetomotive force and the material's non-linear magnetization curve.

\section{Weight optimization}

Since the desired magnetic force is given by the specifications, the question arises at which mean flux density the magnetic bearing should be operated. To come to a conclusion we summarized the optimization results in "figure 6". The following three electrical steels are used:

- steel 1: standard transformer steel (measured data)

- steel 2: high flux cobalt steel, Vacodur 50, optimum magnetic properties [6]

- steel 3: high flux cobalt steel, Vacodur 50, high tensile strength [6]

Every point in figure 6 represents a finished optimization process. They all share the same desired 
magnetic force. The curves are cascade shaped on the left hand side. This underlies the increasing magnetomotive force, which requires more windings since the maximum current is constant. When one winding row is full, the next is filled up. This leads to an abrupt increase of the effective core length, resulting in an abrupt decrease of the force to weight ratio.

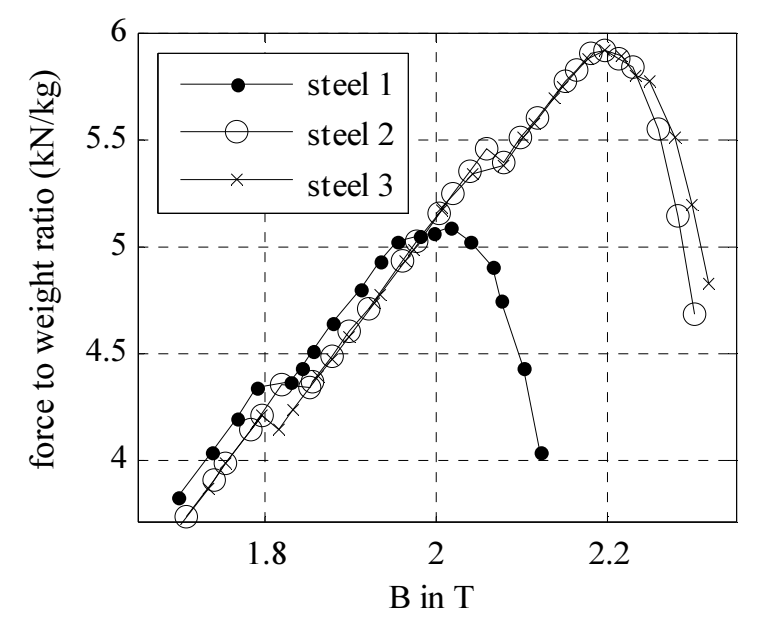

Fig. 6: Force to weight ratio

The optima in "figure 6" (steel 1 with B $=2.0 \mathrm{~T}$ and steel 2 and steel 3 with $\mathrm{B}=2.2 \mathrm{~T}$ ) for the best average magnetic flux density are consistent with the mean B-H diagram of these materials.

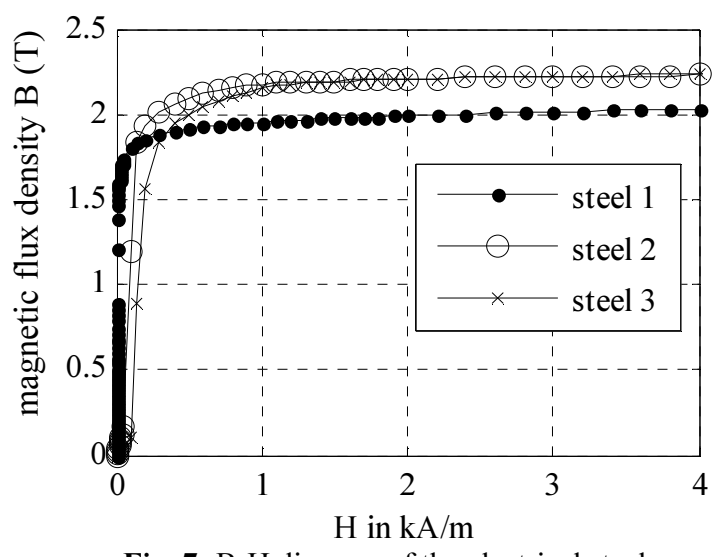

Fig. 7: B-H diagram of the electrical steels

As promising the high flux cobalt steel is, it has some disadvantages. First, it's significantly higher priced, see "figure 8" and secondly it's significantly higher hysteresis losses is a further disadvantage. The coercivity of "steel 2" is about ten times higher than the coercivity of "steel 1", which one can hardly see in "figure 7". Finally the third disadvantage is the about $20 \%$ higher electrical conductivity, which is resulting in higher eddy current losses.

\section{Cost optimization}

The estimated costs are compared in "figure 8". Since mass production prices or prototype prices are difficult to determine, the costs are just a rough estimation of a possible mass production. The material costs are derived from mass production transformers. As magnetic bearings, they are basically constructed by electrical steel and copper windings. The exact position of the optimum is dependent on the ratio of the steel price to the enamelled copper wire price. Varying the ratio by a factor of 10 moves the optimum from $1.95 \mathrm{~T}$ to $2.02 \mathrm{~T}$ for "steel 1". An increase or decrease of the copper and steel price, just moves the curve up and down. However, the optimum remains.

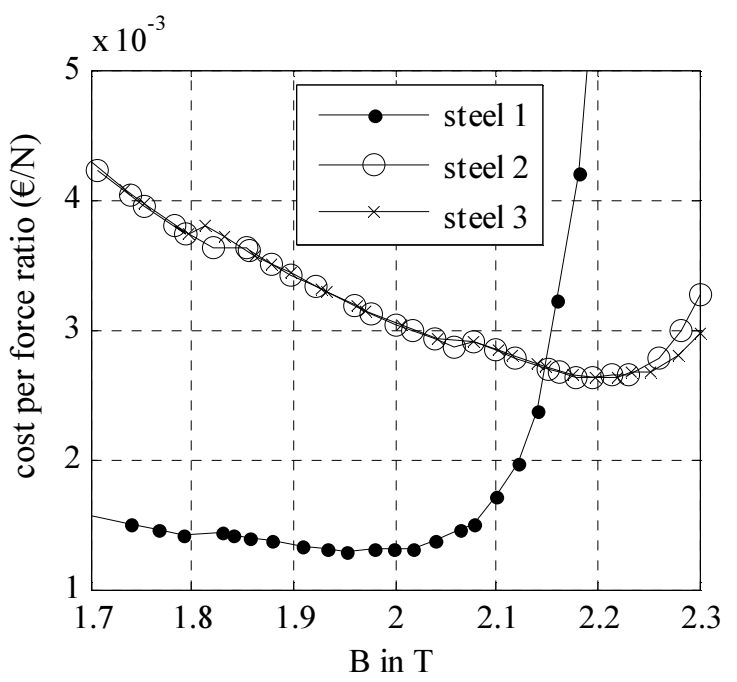

Fig. 8: Estimation of costs

Whether the prices or their ratio is correct, the cost optimum lies in a narrow range. Fortunately the optima of the weight and cost optimization are very close. Based on the knowledge of the last two sections, the desired average flux density is known.

\section{Optimization}

As mentioned in section 1, the cost function is not continuous. Finding the number of windings with the best geometry to get the desired average flux density and the desired magnetic force can be managed with a stochastic approach as shown in [4].

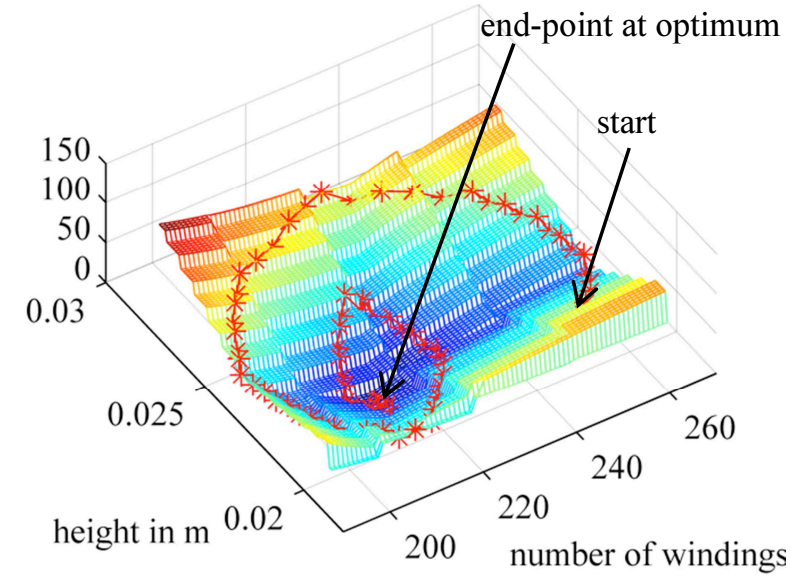

Fig. 9: Optimization of one solution plotted over the noncontinuous cost function

The method used here is a deterministic approach. It is gradient based, but the parameter variation of the number of windings and of the iron cross section is controlled independently. This means there are two cost 
functions. One for the magnetic force and one for the average flux density. Both step sizes are controlled independently by their cost function. Therefore the optimization is not solved in the fastest way, as can be seen in "figure 9", where the optimization process slowly circles into the global optimum. This however is quite irrelevant since the whole process just takes about a second. With this unconventional approach even the discontinuous cost function is not a problem. The step size to change the iron's cross-sectional area and the number of windings does play an important role. The lamination thickness is $0.35 \mathrm{~mm}$ and the copper windings have a cross-sectional area of $0.5 \mathrm{~mm}^{2}$. The air gap is $400 \mu \mathrm{m}$. If there is an additional desired current rise-time and the amplifier's specifications are not yet fixed, the copper wire's cross-sectional area can be included into the optimization process.

\section{Conclusion}

This paper presents a method to find and compare the weight and the cost dependent optimum of magnetic bearings with electrical steel. The optima are fundamentally based on the magnetization curve of the desired magnetic material. The magnetization curve can be extracted from a datasheet or can be obtained by measurements. The presented equivalent circuit model enables to calculate the magnetic force and to compute the average magnetic flux density very quickly. The equivalent circuit model can additionally be adapted by several parameters to gain more accuracy. With this method hundreds of optimization processes can be compared with relatively low calculation effort. This can be done quickly and without the need to compute the cost function with finite element method software. It does require a fitting of the model parameters to the FEM simulation results though.

\section{Acknowledgement}

Our Research has been supported by the Neue Energien 2020 program of the Klima + Energie fonds Grant No. (FFG 825.553).

\section{References}

1. M. Bader, B. Schweighofer, H. Wegleiter, Proceedings of the European Electric Vehicle Conference, (2011)

2. M. Kasarda, P. Allaire, P. Norris, C. Mastrangelo, E. Maslen, J. Eng. Gas Turb. Power, 121, 697-702 (1999)

3. G. Ding, Z. Zhoiu, Y. Hu, D. He, Proceedings of the International Conference on Mechatronic and Embedded Systems and Applications, 1-6 (2006)

4. G. Stumberger, D. Dolinar, U. Pahner, K. Hameyer, IEEE Trans. Magn., 36, 1009-1013 (2000)

5. Y. Sun, Y. Ho, L. Yu, IEEE Trans. Magn., 45, 139149 (2009)

6. Vacoflux/Vacodur, datasheet VACUUMSCHMELZE (2004) 